\title{
First Candle Forming Public Health Advisory Committee to Address Health Inequities
}

Barb Himes, IBCLC

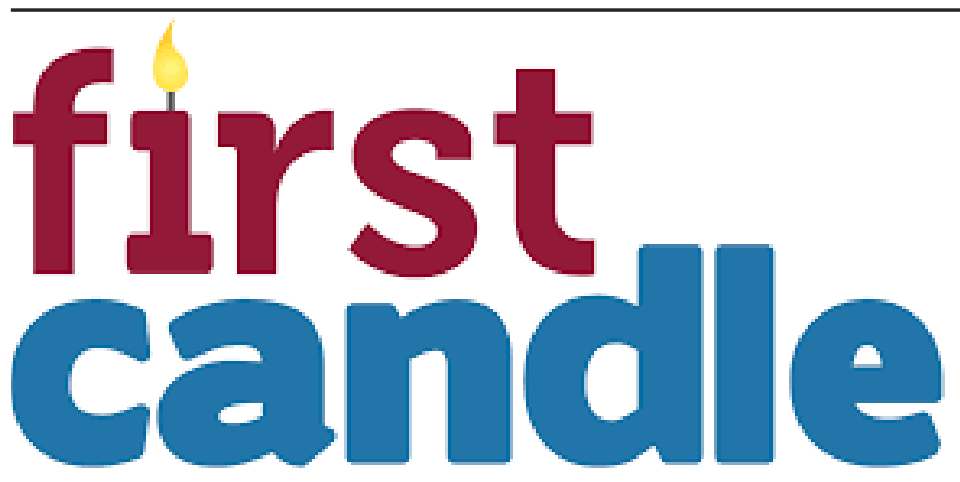

Saving babies. Supporting families.

First Candle's efforts to support families during their most difficult times and provide new answers to help other families avoid the tragedy of the loss of their baby are without parallel.

"We have previously shared information with Neonatology Today readers about our work to lower the rates of sleep-related infant deaths in the U.S., first as a charter participant in the 1994 national Back to Sleep (now Safe to Sleep) campaign, which contributed to a $50 \%$ reduction in the SIDS rate, and our ongoing educational outreach to professionals and families. "

We have previously shared information with Neonatology Today readers about our work to lower the rates of sleep-related infant deaths in the U.S., first as a charter participant in the 1994 national Back to Sleep (now Safe to Sleep) campaign, which contributed to a $50 \%$ reduction in the SIDS rate, and our ongoing educational outreach to professionals and families.

At the core of this has been the set of safe sleep guidelines developed by the American Academy of Pediatrics (AAP), which continues to be updated and which forms the foundation of our own Straight Talk for Infant Safe Sleep training program targeted to health care professionals who provide maternal and infant health services to families.

Through our work, we have come face to face with the complexities that affect parents' adoption of the guidelines, with the perseverance required to create long-term change in health behaviors; and the larger challenges within the health care system that families depend on for maternal and infant health. We have seen that a thread running through all of this is implicit bias and its impact on maternal and infant health and mortality in the U.S.

Presenting evidence-based safe sleep guidelines is not enough; the context in which it is conveyed to health care professionals and families has a direct bearing on how it is received, accepted, or rejected.

Through our Straight Talk for Infant Safe Sleep, we have been helping health care professionals identify implicit bias and learn how to address it. In Straight Talk and our overall organizational work, we look at bias in the many forms it takes, from gender to socioeconomic, from deskside manner to race, from small interpersonal behaviors to institutional practices.

But there is always more to be done. Today the rate of SUID among black infants is three times higher than for whites, and for Hispanic babies, it is twice as high. And while the AAP guidelines recommend regular prenatal care for pregnant women, maternal health in communities of Color is threatened by health inequities resulting from structural racism in health care, lack of access to quality prenatal and postpartum care, and racial stress. These can contribute to low birth weight, pre-term birth and birth complications, all infant death risk factors.

Therefore, we are expanding our efforts and are developing a standing Public Health Advisory Committee of health care professionals and advocates to constructively address the racial disparity, health inequity, and implicit bias in maternal and infant health practices and their impacts on sleep-related infant mortality. This committee will also help us ensure that our educational outreach messages and materials work effectively in the real world that families experience.

This volunteer committee will include leaders ranging from health care and public health outreach professionals to national maternal justice advocacy organizations and policymakers. Founding members include First Candle board members Andrew Rubenstein, MD, Chair, Obstetrics and Gynecology for the Dignity Health Medical Group at Saint Joseph's Hospital and Medical Center in Phoenix; and John Ciannella, MD,

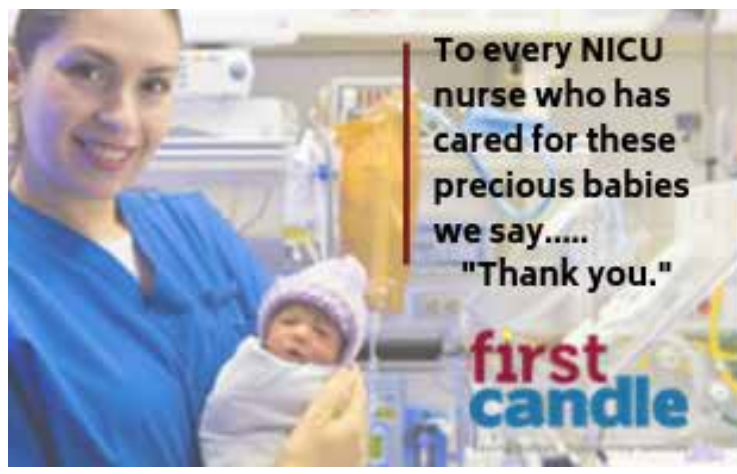

\section{Did you know that premature and low birth weight babies have a $4 \times$ greater risk for SIDS?}

At First Candle we're educating parents, grandparents and caregivers about safer sleep to make sure all babies reach their first birthday. Learn more at firstcandle.org 


\section{"This volunteer committee will include leaders ranging from health care and public health outreach professionals to national maternal justice advocacy organizations and policymakers."}

Dr. Rubenstein considers "the formation of this diverse, multi-disciplinary advisory committee paramount to drive changes within the healthcare models and address the disparities in maternal and child health."

Another member is Dauline Singletary, MPH, Public Health Educator at Wake County Health in Durham, North Carolina, and a Doula, who notes that "working in public health, we all see that the racial and health inequities in Black and brown communities are much higher than that of white communities, and developing a committee that focuses on the needs and providing the resources not only promotes health babies but healthy families."

The committee-in-formation is also expected to include representatives from organizations focused on inequities in maternal morbidity rates as well, and to look at premature and low birth weight births, where infants have a four times greater risk of SIDS.

This committee formation complements our recent increased public outreach around family perceptions of the AAP safe sleep guidelines. We have been convening community task forces in Georgia, Connecticut, and Michigan, comprised of parents, extended family, in-home health care providers, social service agencies, and doulas, to learn their thoughts on the guidelines and the challenges in adopting them, as well as the role systemic racism plays.

The findings are being shared with the AAP as it develops the updated safe sleep guidelines, scheduled to be released later in 2021. The community task force participants will then have the opportunity to be involved in framing how the revised guidelines are communicated to

\section{"The committee-in-formation is also} expected to include representatives from organizations focused on inequities in maternal morbidity rates as well, and to look at premature and low birth weight births, where infants have a four times greater risk of SIDS."

their specific communities.

We want every baby to reach his or her first birthday and beyond. Health inequities imperil this, but the answers lie within the relationship between health care providers and families, and we feel it is our job to listen, learn and provide both leadership and support.

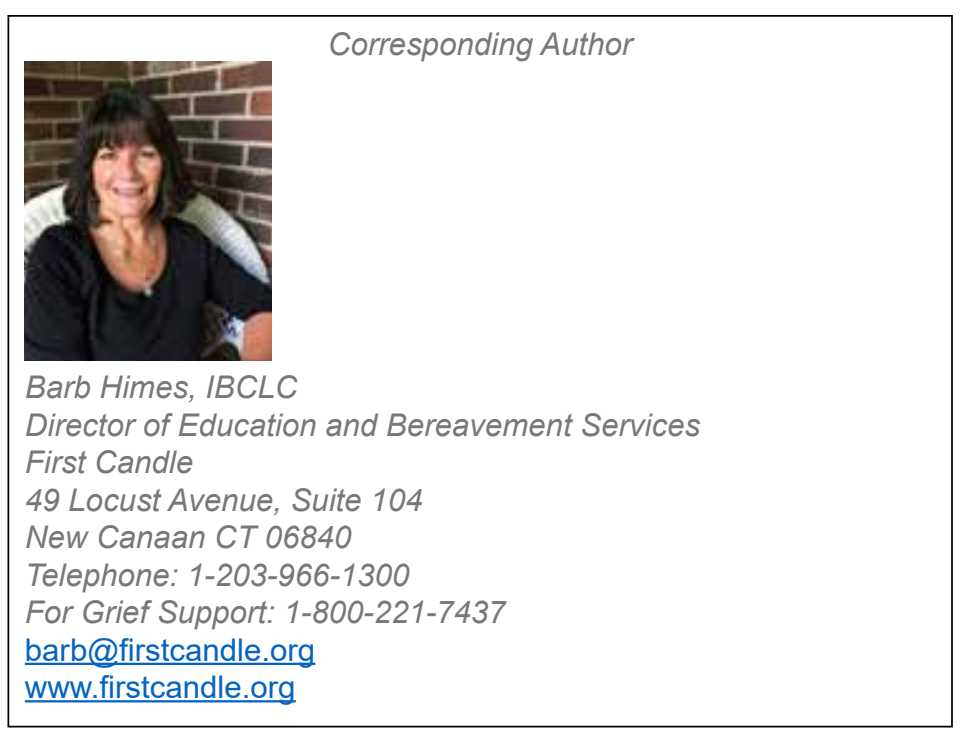

\section{About First Candle}

First Candle, based in New Canaan, CT, is a 501c (3) committed to eliminating Sudden Infant Death Syndrome and other sleep-related infant deaths while providing bereavement support for families who have suffered a loss. Sudden unexpected infant death (SUID), which includes SIDS and accidental suffocation and strangulation in bed (ASSB), remains the leading cause of death for babies one month to one year of age, resulting in 3,600 infant deaths nationwide per year.

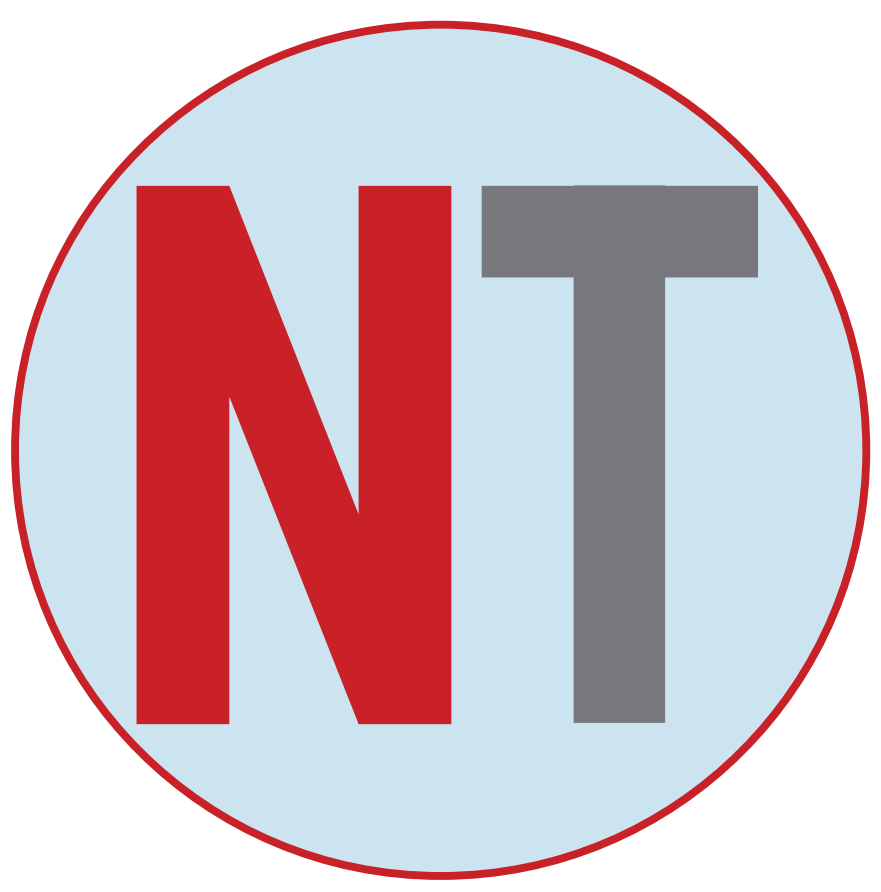

